laboratory and management characteristics along with the clinical course of Budd-Chiari syndrome (BCS) associated with Behçet's disease (BD).

Methods: Sixty patients with BD with BCS (40 male, 20 female) were identified in 23 rheumatology centers (Group I). A total of 169 consecutive patients (100 male, 69 female) with BD who did not have clinically apparent BCS during the follow-up were evaluated as the control group (Group II).

Results: Comparison of the demographic and clinical findings between the Group I and the Group II were as follows: The mean age of disease onset was $23.1+$-/ 6.7 years vs. $26.8 \pm 0.6$ years ( $p=0.013$ ), mean age at diagnosis was $27.2 \pm 0.9$ vs. $30.4 \pm 0.6$ years $(\mathrm{p}=0.008)$, arthritis was $10 \%$ vs. $28.4 \%(\mathrm{p}=0.002)$, papulopustular skin lesion was $48.3 \%$ vs $69.2 \%(p=0.003)$, central nervous system (CNS) involvement $10 \%$ vs. $3 \%(p=0.03)$, cardiac involvement was $16.7 \%$ vs. $2.4 \%$ $(p<0.001)$, superficial thrombophlebitis was $23.3 \%$ vs. $4.7 \%(p<0.001)$, and deep vein thrombosis was $58.3 \%$ vs. $15.4 \%(p<0.01)$. On diagnosis $50 \%$ of BD patients with BCS were classified as Child-Pugh A. Inferior vena cava obstruction was observed in $38.3 \%$ and portal vein thrombosis was seen in $3.3 \%$ of the patients with BCS. Mortality in BCS patients with BD was $18.3 \%$. BCS related treatment after diagnosis in patients with BD were as follows: $71.7 \%$ of patients were treated with monthly cyclophosphamide intravenous pulses, $53.3 \%$ received intravenous pulse corticosteroids, $55.9 \%$ used azathioprine, $54.2 \%$ had warfarine treatment, and $50.8 \%$ were treated with low molecular weight heparin.

Conclusions: This study shows a higher frequency of cardiac and CNS involvement, superficial thrombophlebitis, papulopustular skin lesion, deep vein thrombosis in BD patients with BCS. Arthritis was observed less common in BD patients with BCS. The mean age onset was lower in patients with BCS. Medical treatment with immunosuppressive agents and anticoagulation appears to be the treatment of choice in BD patients with BCS. The majority of the patients with BCS were Child-Pugh class A on diagnosis. The inferior vena cava is frequently involved and, often associated with deep vein thrombosis and cardiac involvement.

Disclosure of Interest: None declared

DOI: 10.1136/annrheumdis-2017-eular.3559

\section{THU0330 MYCOBACTERIAL CORD FACTOR ANALOG INDUCES HIGH IL-6 SECRETION AND MINCLE RECEPTOR EXPRESSION IN PATIENTS WITH TAKAYASU ARTERITIS}

N. Gupta ${ }^{1}$, J. Kabeer Doss ${ }^{2}$, H. Mohan ${ }^{2}$, R. Goel ${ }^{2}$, D. Danda ${ }^{2} .{ }^{1}$ CHRistian Medical College, VELLORE, Vellore, India; ${ }^{2}$ Clinical Immunology \& Rheumatology, CHRistian Medical College, VELLORE, Vellore, India

Background: Association between Takayasu Arteritis (TA) and Tubcerculosis (TB) has been in vogue for years. Scientific evidence for this association is limited. Objectives: To study pro-inflammatory cytokine responses and mRNA expression in patients with TA on exposure to Trehalose-6,6-dibehenate (TDB), a synthetic analogue of mycobacterial cord factor in comparison with healthy controls.

Methods: Patients with type V TA satisfying 1990 ACR criteria and age and sex matched healthy controls were recruited. $10 \mathrm{ml}$ blood was collected in heparin coated tube. PBMCs were separated by density gradient centrifugation. PBMCs were cultured with and without $5 \mu \mathrm{g} / \mathrm{ml}$ and/or $50 \mu \mathrm{g} / \mathrm{ml}$ of TDB for 48 hours in RPMI medium at $5 \%$ co2 incubator. IL-6, TNF- $\alpha$ and IL-17 were measured in cell culture supernatant. RNA was extracted from the cells and quantification of gene expression was performed using sequence specific primers with SYBR green in Step One Plus ${ }^{\text {TM }}$ Real-Time PCR Systems (Applied Biosystems).

Results: Twenty two TA patients and 21 age and sex matched healthy controls were recruited. Baseline characteristic of cases included median (range) age:26.5 years (15-49), male:femaleratio of 5:17, ITAS-CRP2010: 7 (1-26) and DEITAK: 7.5 (1-21). Both patients and controls showed response by secreting IL- 6 and TNF- $\alpha$ upon stimulation withTDB. Relative induction of IL- 6 was significantly higher in TA [31.88 (0.74-168)] as compared to healthy controls [1.931 (0.644-8.21); $\mathrm{p}<0.002$ ]. IL-17 was undetectable even upon TDB stimulation. Relative mincle expression was significantly upregulated by TDB in TA1.03 (0.623-1.346) fold, as compared to healthy controls $0.43(0.36-0.57)$ fold $(p<0.05)$. Relative gene expressions of IL-6, IL-8, TNF $\alpha$, IFN-gand BCL-10 were not significantly different between TA and healthy controls.

Conclusions: Stimulation with TDB led to higher secretion of IL- 6 and increased mincle expression in PBMCs of TA patients as compared to healthy controls.

Disclosure of Interest: None declared

DOI: 10.1136/annrheumdis-2017-eular.6068

\section{THU0331 MYELOPEROXIDASE DEPLETION IS A SELECTIVE FEATURE OF TAKAYASU ARTERITIS AND PREDICTS THE CLINICAL OUTCOME}

A.A. Manfredi, G.A. Ramirez, V. Canti, E. Tombetti, M. Baldini, E. Baldisera, P. Rovere-Querini, N. Maugeri. San Raffaele Scientific Institute, Milano, Italy

Background: Takayasu's arteritis (TA) is a rare large-vessel vasculitis characterized by persistent remodelling of the vessel walls. The role of innate immune cells in TA is poorly understood. Biomarkers to be used for assessment of TA activity and clinical outcome are missing. A trimodal pattern of myeloperoxidase (MPO) distribution (simultaneous occurrence of neutrophils with complete depletion, reduced and normal content of the enzyme) is detectable in the very early phase of acute myocardial infarction and has been associated with a burst of neutrophil interaction with activated platelets ${ }^{1}$

Objectives: Here, we aimed at characterizing the phenotype of neutrophils and platelets in patients TA and correlating these biological findings with clinical data. Methods: neutrophil MPO expression has been studies in 93 subjects, including 21 TA patients, 20 age- and sex-matched healthy donors that served as controls, 25 patients with chronic stable atherosclerosis (CSA), eight patients with giant cell arteritis (GCA), five patients with gramulomatosis with polyangitis (GPA), four patients with eosinophilic GPA (EGPA) and ten patients with polymyalgia rheumatica (PMR). Blood samples were collected and processed as described ${ }^{1-3}$ Neutrophil MPO content, platelet P-selectin and High-Mobility Group B1 (HMGB1) in platelet derived microparticles $(\mathrm{PD} \mu \mathrm{P})$ release were assessed by flow cytometry. TA patients were then followed up for a median time of 3.43 years.

Results: The trimodal distribution of neutrophils MPO content was identified in 17/21 TA, 1/8 GCA, 0/10 PMR, 0/25 CSA, 0/5 GPA and 0/4 EGPA patients; moreover in $0 / 20$ healthy controls (ANOVA, $F=15.22, p<0.0001$ ). Neutrophil MPO content and the presence of neutrophils with complete MPO depletion in TA patients correlated inversely with i) platelet $P$-selectin expression $(p<0.01)$, ii) the fraction of HMGB1 ${ }^{+}-\mathrm{PD} \mu \mathrm{P}(\mathrm{p}<0.01)$ and iii) disease duration, implicating an association of platelet and neutrophil activation with the disease natural history. In fact, the Kaplan Mayer analysis reveals that TA patients that had neutrophils with complete MPO depletion are at a significantly increased risk of ischemic complications during the follow up (long-rank=4.864, $\mathrm{p}=0.027, \mathrm{HR}=36.15$ abd $\mathrm{Cl}-95 \%=0.15-7.51$ )

Conclusions: Neutrophils and platelets are significantly activated in TA. Parox ysmal neutrophils activation, leading to complete MPO depletion, is a common feature and predict ischemic events and as such the clinical disease outcome.

References:

[1] Maugeri N, Rovere-Querini P, Evangelista V, et al. An intense and short-lasting burst of neutrophil activation differentiates early acute myocardial infarction from systemic inflammatory syndromes. PLoS One 2012;7:e39484.

[2] Manfredi AA, Baldini M, Camera M, et al. Anti-TNFalpha agents curb platelet activation in patients with rheumatoid arthritis. Annals of the rheumatic diseases 2016;75:1511-20.

[3] Maugeri N, Campana L, Gavina M, et al. Activated platelets present high mobility group box 1 to neutrophils, inducing autophagy and promoting the extrusion of neutrophil extracellular traps. Journal of thrombosis and haemostasis: JTH 2014;12:2074-88.

Disclosure of Interest: None declared

DOI: 10.1136/annrheumdis-2017-eular.4940

\section{THU0332 CARDIAC INVOLVEMENT IN POLYARTERITIS NODOSA: A RETROSPECTIVE PATHOLOGICAL STUDY OF 37 AUTOPSY CASES}

O. Zimba ${ }^{1}$, M. Bagriy ${ }^{2} .{ }^{1}$ the Department of Internal Medicine, Danylo Halytsky Lviv National Medical University, Lviv; ${ }^{2}$ Department of Pathological Anatomy, Ivano-Frankivsk National Medical University, Ivano-Frankivsk, Ukraine

Background: Cardiac involvement is a significant cause of the death and disability in systemic vasculitis. The comprehensive analysis of histopathologic findings in polyarteritis nodosa (PN) can help better understand mechanisms of excessive cardiovascular risk in patients with systemic vasculitis.

Objectives: To investigate the variation of cardiac pathological findings in autopsy cases of PN.

Methods: A retrospective analysis of cardiac pathological changes was performed in 37 autopsy cases of PN over period of 15 years. There were 28 males and 9 females, ranging from 23 to 85 years. The median age at disease onset was 35 , 7 years; the median duration of disease was 2.1 years with a range of 2 month to 7 years.

Results: The destructive-productive vasculitis of the coronary arteries have been found in 30 cases $(81.1 \%)$ of PN. The vessels most affected were myocardial, and epicardial medium and small-sized arteries. Histologically, the remodelling of coronary artery tree in PN had a wide range of acute and chronic changes that likely were depended on the duration of the disease. There were three types of pathological findings: (1) arteritis with predominantly destructive changes; (2) arteritis with predominantly proliferative changes; (3) destructive-productive arteritis. The early phase of PN has been characterized by abnormalities of vessels wall from mucoid swelling to fibrinoid necrosis. The most common was panarteritis with involvement of all layers of the arterial wall, and inflammatory responses with intramural, and perivascular infiltrates, mainly composed of lymphocytes, and neutrophils. However, endo-, meso-, and periarteritis also were observed in some cases. The most remarkable lesions in coronary arteries were the nodules visible to the naked eye along coronary arteries. They were present in 9 cases $(30 \%)$, and can be described as well-demarcated nodular thickening of the artery walls with size from 0.1 to $0.4 \mathrm{~cm}$. The pathological basis of nodules are areas of focal inflammation, destruction and aneurysmal dilatations of artery wall. In cases of chronic course of PN, the intimal hyperplasia due to proliferation of endothelial cells have been observed. Interestingly, that this productive endarteritis with luminal narrowing of coronary arteries was common in patients who were exposed to different types of occupational xenobiotic (silica dust, pesticides, insecticides, solvents, heavy metals). In 5 (16.7\%) cases, luminal occlusion due to intimal proliferation, fibrosis and thrombus formation 